\title{
Differential Subordinations and Harmonic Means
}

\author{
Stanisława Kanas • Andreea-Elena Tudor
}

Received: 9 January 2014 / Revised: 3 April 2014 / Published online: 22 January 2015

(c) Malaysian Mathematical Sciences Society and Universiti Sains Malaysia 2015

\begin{abstract}
The aim of a study of the presented paper is the differential subordination involving harmonic means of the expressions $p(z), p(z)+z p^{\prime}(z)$, and $p(z)+\frac{z p^{\prime}(z)}{p(z)}$ when $p$ is an analytic function in the unit disk, such that $p(0)=1, p(z) \not \equiv 1$. Several applications in the geometric functions theory are given.
\end{abstract}

Keywords Univalent functions - Subordination - Differential subordination · Harmonic means

Mathematics Subject Classification Primary 31A05 - Secondary 30C45

\section{Introduction}

The harmonic mean, known also as the subcontrary mean, is one of several kinds of average, and is the special case power mean. It is used for the situations when the average of rates is desired and has several applications in geometry, trigonometry, probabilistics and statistics, algebra, physics, finance, computer science, etc. The harmonic mean is one of the Pythagorean means, along with the arithmetic and the geometric mean, and is no greater than either of them. The harmonic mean $H$ of

Communicated by V. Ravichandran.

S. Kanas $(\bowtie)$

University of Rzeszow, ul. S. Pigonia 1, 35-310 Rzeszow, Poland

e-mail: skanas@ur.edu.pl

A.-E. Tudor

"Babeş-Bolyai" University, 1 Kogălniceanu Street, 400084 Cluj-Napoca, Romania

e-mail: tudor_andreea_elena@yahoo.com 
the positive real numbers $x_{1}, x_{2}, \ldots, x_{n}$ is defined to be the reciprocal of the arithmetic mean of the reciprocals of $x_{1}, x_{2}, \ldots, x_{n}$

$$
H=\left(\frac{1}{n} \sum_{k=1}^{n} \frac{1}{x_{k}}\right)^{-1} .
$$

For the special case of just two numbers $x_{1}$ and $x_{2}$, the harmonic mean can be written

$$
H=\frac{2 x_{1} x_{2}}{x_{1}+x_{2}} .
$$

In this special case, the harmonic mean is related to the arithmetic mean

$$
A=\frac{x_{1}+x_{2}}{2}, \text { and the geometric mean } G=\sqrt{x_{1} x_{2}},
$$

by $H=\frac{G^{2}}{A}$, or $G=\sqrt{A H}$ meaning the two numbers' geometric mean equals the geometric mean of their arithmetic and harmonic means.

Several concepts of the classes involving arithmetic and geometric means were appeared in the literature (see e.g. [1-9,11-14,16,17], for the extensive studies, we refer to the Miller and Mocanu monograph [10]).

The purpose of this paper is to study the harmonic mean, as a supplementary to the well-known arithmetics and geometric Pythagorean means. In addition, a new mean brings along a wide range of new possibilities for exploiting harmonic ideas in connection of several quantities or functionals in the geometric function theory.

In order to prove our main results, we introduce some fundamental notions and notations.

Let $\mathcal{A}$ be the class of all analytic functions $f$ in the open unit disk $\mathbb{D}=$ $\{z \in \mathbb{C}:|z|<1\}$, of the form:

$$
f(z)=z+\sum_{k=2}^{\infty} a_{k} z^{k}
$$

If $f$ and $g$ are two functions analytic in $\mathbb{D}$, we say that $f$ is subordinate to $g$, written as $f \prec g$ or $f(z) \prec g(z)$, if there exists a Schwarz function $\omega$ (i.e., analytic in $\mathbb{D}$, with $\omega(0)=0$ and $|\omega(z)|<1$, for all $\mathbb{D})$ such that $f(z)=g(\omega(z)), z \in \mathbb{D}$. Furthermore, if $g$ is univalent in $\mathbb{D}$, then we have the following equivalence:

$$
f(z) \prec g(z) \Leftrightarrow f(0)=g(0) \text { and } f(\mathbb{D}) \subset g(\mathbb{D}) .
$$

Definition 1.1 [10, p. 21] Denote by $Q$ the set of functions $q$ that are analytic and injective on $\overline{\mathbb{D}} \backslash E(q)$, where

$$
E(q)=\left\{\zeta \in \partial \mathbb{D}: \lim _{z \rightarrow \zeta} q(z)=\infty\right\},
$$


such that $q^{\prime}(\zeta) \neq 0$ for $\zeta \in \partial \mathbb{D} \backslash E(q)$.

If $q \in Q$ then $\Delta=q(\mathbb{D})$ is a simply connected domain.

In order to prove our main results, we will need the following lemma:

Lemma $1.1\left[10\right.$, p. 24] Let $q \in Q$, with $q(0)=a$, and let $p(z)=a+a_{n} z^{n}+\cdots$ be analytic in $\mathbb{D}$ with $p(z) \not \equiv a$ and $n \geq 1$. If $p$ is not subordinate to $q$, then there exist points $z_{0}=r_{0} e^{i \theta_{0}} \in \mathbb{D}$ and $\zeta_{0} \in \partial \mathbb{D} \backslash E(q)$ and an $m \geq n \geq 1$ for which $p\left(\mathbb{D}_{r_{0}}\right) \subset q(\mathbb{D})$,

(1) $p\left(z_{0}\right)=q\left(\zeta_{0}\right)$,

(2) $z_{0} p^{\prime}\left(z_{0}\right)=m \zeta_{0} q^{\prime}\left(\zeta_{0}\right)$,

(3) $\Re \frac{z_{0} p^{\prime \prime}\left(z_{0}\right)}{p^{\prime}\left(z_{0}\right)}+1 \geq m \Re\left[\frac{\zeta_{0} q^{\prime \prime}\left(\zeta_{0}\right)}{q^{\prime}\left(\zeta_{0}\right)}+1\right]$.

\section{Harmonic Mean}

Let $p(z)=1+a_{1} z+\cdots$ be analytic in $\mathbb{D}$ with $p(z) \not \equiv 1$. Then $p(z)+z p^{\prime}(z), p(z)+$ $\frac{z p^{\prime}(z)}{p(z)}$ has the same normalization and play an important role in the theory of differential subordination. Let $f \in \mathcal{A}$ and set $p(z)=\frac{f(z)}{z}$. Then $p(z)+z p^{\prime}(z)=f^{\prime}(z)$, and if $p(z)=\frac{z f^{\prime}(z)}{f(z)}$, then $p(z)+\frac{z p^{\prime}(z)}{p(z)}=1+\frac{z f^{\prime \prime}(z)}{f^{\prime}(z)}$. Extremal properties of such expressions, as well as several relations, were frequently considered in the theory of univalent functions. In this section, we study the differential subordination involving harmonic means of such expressions, and present some applications in the geometric functions theory.

Theorem 2.1 Let $p(z)=1+a_{1} z+\cdots$ be analytic in $\mathbb{D}$ with $p(z) \not \equiv 1$. Then

$$
\Re\left\{\frac{2 p(z)\left[p(z)+z p^{\prime}(z)\right]}{2 p(z)+z p^{\prime}(z)}\right\}>0 \Rightarrow \Re p(z)>0 .
$$

Proof Let

$$
q(z)=\frac{1+z}{1-z}=1+q_{1} z+\cdots,
$$

with $\Delta=q(\mathbb{D})=\{w: \Re w>0\}, q(0)=1, E(q)=\{1\}$ and $q \in Q$. Then, we can rewrite the condition $(2.1)$ as

$$
\Re\left\{\frac{2 p(z)\left[p(z)+z p^{\prime}(z)\right]}{2 p(z)+z p^{\prime}(z)}\right\}>0 \Rightarrow p(z) \prec q(z) .
$$

Suppose that $p(z) \nprec q(z)$. Then, from Lemma 1.1 , there exist a point $z_{0} \in \mathbb{D}$ and a point $\zeta_{0} \in \partial \mathbb{D} \backslash\{1\}$ such that $p\left(z_{0}\right)=q\left(\zeta_{0}\right)$ and $\Re p(z)>0$ for all $z \in \mathbb{D}_{\left|z_{0}\right|}$. This implies that $\Re p\left(z_{0}\right)=0$, therefore we can choose $p\left(z_{0}\right)$ of the form $p\left(z_{0}\right):=i x$, 
where $x$ is a real number. Due to symmetry, it is sufficient to consider only the case where $x>0$. We have

$$
\zeta_{0}=q^{-1}\left(p\left(z_{0}\right)\right)=\frac{p\left(z_{0}\right)-1}{p\left(z_{0}\right)+1}
$$

then $z_{0} p^{\prime}\left(z_{0}\right)=m \zeta_{0} q^{\prime}\left(\zeta_{0}\right)=-m\left(x^{2}+1\right) / 2:=y$, where $y<0$.

Thus, we obtain:

$$
\Re\left\{\frac{2 p\left(z_{0}\right)\left[p\left(z_{0}\right)+z_{0} p^{\prime}\left(z_{0}\right)\right]}{2 p\left(z_{0}\right)+z_{0} p^{\prime}\left(z_{0}\right)}\right\}=\Re\left[\frac{2 i x(i x+y)}{2 i x+y}\right]=\frac{2 x^{2} y}{4 x^{2}+y^{2}}<0 .
$$

This contradicts the hypothesis of the theorem, therefore $p \prec q$ and the proof of Theorem 2.1 is complete.

Remark 1 We only note that the expression of the left hand side of (2.1) is of the harmonic form of two elements $x_{1}=p(z)$ and $x_{2}=p(z)+z p^{\prime}(z)(z \in \mathbb{D})$.

Setting $p(z)=\frac{f(z)}{z}$ in the previous theorem, we obtain the following corollary:

Corollary 2.1 Let $f(z)=z+a_{2} z^{2}+\cdots$ be analytic in $\mathbb{D}$. Then

$$
\Re \frac{2 f(z) f^{\prime}(z)}{f(z)+z f^{\prime}(z)}>0 \Rightarrow \Re \frac{f(z)}{z}>0 .
$$

Theorem 2.2 Let $p(z)=1+a_{1} z+\cdots$ be analytic in $\mathbb{D}$ with $p(z) \not \equiv 1$. Then

$$
\Re\left[\frac{2 p(z)+2 z p^{\prime}(z)}{1+p^{2}(z)+z p(z) p^{\prime}(z)}\right]>0 \Rightarrow \Re p(z)>0 .
$$

Proof Following the same steps as in the proof of Theorem 2.1, setting $p\left(z_{0}\right)=$ $i x, x>0$ and $z_{0} p^{\prime}\left(z_{0}\right)=y, y<0$, we obtain:

$\Re\left[\frac{2 p\left(z_{0}\right)+2 z_{0} p^{\prime}\left(z_{0}\right)}{1+p^{2}\left(z_{0}\right)+z_{0} p\left(z_{0}\right) p^{\prime}\left(z_{0}\right)}\right]=\Re\left[\frac{2 i x+2 y}{1-x^{2}+i x y}\right]=\frac{2 y}{\left(1-x^{2}\right)^{2}+x^{2} y^{2}}<0$,

which completes the proof.

Setting $p(z)=\frac{f(z)}{z}$ we obtain

Corollary 2.2 Let $f(z)=z+a_{2} z^{2}+\cdots$ be analytic in $\mathbb{D}$. Then

$$
\Re\left[\frac{2 \frac{z}{f(z)} f^{\prime}(z)}{\frac{z}{f(z)}+f^{\prime}(z)}\right]>0 \Rightarrow \Re \frac{f(z)}{z}>0 .
$$


Theorem 2.3 Let $p(z)=1+a_{1} z+\cdots$ be analytic in $\mathbb{D}$ with $p(z) \not \equiv 1$. Then

$$
\Re\left\{\frac{2\left[p(z)+\frac{z p^{\prime}(z)}{p(z)}\right]}{2+\frac{z p^{\prime}(z)}{p^{2}(z)}}\right\}>0 \Rightarrow \Re p(z)>0 .
$$

Proof We only need to show that $p \prec q$, where $q$ is given by (2.2). As in the proof of Theorem 2.1, there exist a point $z_{0} \in \mathbb{D}$ such that $p\left(z_{0}\right)=i x, x>0$ and $z_{0} p^{\prime}\left(z_{0}\right)=y, y<0$. Therefore, we have

$$
\Re\left\{\frac{2\left[p\left(z_{0}\right)+\frac{z_{0} p^{\prime}\left(z_{0}\right)}{p\left(z_{0}\right)}\right]}{2+\frac{z_{0} p^{\prime}\left(z_{0}\right)}{p^{2}\left(z_{0}\right)}}\right\}=\Re\left[\frac{2\left(i x+\frac{y}{i x}\right)}{2-\frac{y}{x}}\right]=0<0 .
$$

and the proof of Theorem 2.3 is completed.

Setting $p(z)=\frac{z f^{\prime}(z)}{f(z)}$ we obtain

Corollary 2.3 Let $f(z)=z+a_{2} z^{2}+\cdots$ be analytic in $\mathbb{D}$. Then

$$
\Re\left\{\frac{2 \frac{z f^{\prime}(z)}{f(z)}\left[1+\frac{z f^{\prime \prime}(z)}{f^{\prime}(z)}\right]}{1+\frac{z f^{\prime}(z)}{f(z)}+\frac{z f^{\prime \prime}(z)}{f^{\prime}(z)}}\right\}>0 \Rightarrow \Re \frac{z f^{\prime}(z)}{f(z)}>0 .
$$

Theorem 2.4 Let $p(z)=1+a_{1} z+\cdots$ be analytic in $\mathbb{D}$ with $p(z) \not \equiv 1$. Then

$$
\Re\left\{\frac{2\left[p(z)+\frac{z p^{\prime}(z)}{p(z)}\right]}{1+p^{2}(z)+z p^{\prime}(z)}\right\}>0 \Rightarrow \Re p(z)>0 .
$$

Proof As in the proof of Theorem 2.1, there exist a point $z_{0}$ in $\mathbb{D}$ such that $p\left(z_{0}\right)=$ $i x, x>0$ and $z_{0} p^{\prime}\left(z_{0}\right)=y, y<0$. We have:

$$
\Re\left\{\frac{2\left[p\left(z_{0}\right)+\frac{z_{0} p^{\prime}\left(z_{0}\right)}{p\left(z_{0}\right)}\right]}{1+p^{2}\left(z_{0}\right)+z_{0} p^{\prime}\left(z_{0}\right)}\right\}=\Re \frac{2\left(i x+\frac{y}{i x}\right)}{1-x^{2}+y}=0,
$$

which completes the proof.

$$
\text { Setting } p(z)=\frac{z f^{\prime}(z)}{f(z)} \text { we obtain }
$$


Corollary 2.4 Let $f(z)=z+a_{2} z^{2}+\cdots$ be analytic in $\mathbb{D}$. Then

$$
\Re\left\{\frac{2 \frac{f(z)}{z f^{\prime}(z)}\left[1+\frac{z f^{\prime \prime}(z)}{f^{\prime}(z)}\right]}{1+\frac{f(z)}{z f^{\prime}(z)}+\frac{z f^{\prime \prime}(z)}{f^{\prime}(z)}}\right\}>0 \Rightarrow \Re \frac{z f^{\prime}(z)}{f(z)}>0 .
$$

Theorem 2.5 Let $p(z)=1+a_{1} z+a_{2} z^{2}+\cdots$ be analytic in $\mathbb{D}$ with $p(z) \not \equiv 1$, and let $0<M<\frac{1}{3}$. Then

$$
\left|\frac{2 p(z)\left[p(z)+z p^{\prime}(z)\right]}{2 p(z)+z p^{\prime}(z)}-1\right|<M \Rightarrow|p(z)-1|<M .
$$

Proof Let

$$
q(z)=1+M z
$$

with $\Delta=q(\mathbb{D})=\{w:|w-1|<M\}, q(0)=1, E(q)=\emptyset$ and $q \in Q$. Then, the condition (2.3) can be rewritten as

$$
\left|\frac{2 p(z)\left[p(z)+z p^{\prime}(z)\right]}{2 p(z)+z p^{\prime}(z)}-1\right|<M \Rightarrow p(z) \prec q(z) .
$$

Suppose that $p(z) \nprec q(z)$.Then, from Lemma 1.1, there exist $z_{0} \in \mathbb{D}, \zeta_{0} \in \partial \mathbb{D}$ and $m \geq 1$ such that $p\left(z_{0}\right)=q\left(\zeta_{0}\right)$ and $\left|p\left(z_{0}\right)-1\right|<M$ for all $z \in \mathbb{D}_{\left|z_{0}\right|}$. This implies that $\left|p\left(z_{0}\right)-1\right|=\left|q\left(\zeta_{0}\right)-1\right|=M$, therefore we can choose $p\left(z_{0}\right)$ of the form $p\left(z_{0}\right):=1+M e^{i \theta}$, where $\theta$ is a real number. We have

$$
\zeta_{0}=q^{-1}\left(p\left(z_{0}\right)\right)=\left[p\left(z_{0}\right)-1\right] / M,
$$

then

$$
z_{0} p^{\prime}\left(z_{0}\right)=m \zeta_{0} q^{\prime}\left(\zeta_{0}\right)=m M e^{i \theta}, \quad m \geq 1 .
$$

We can write:

$$
\begin{aligned}
\left|\frac{2 p\left(z_{0}\right)\left[p\left(z_{0}\right)+z_{0} p^{\prime}\left(z_{0}\right)\right]}{2 p\left(z_{0}\right)+z_{0} p^{\prime}\left(z_{0}\right)}-1\right| & =\left|\frac{2 p^{2}\left(z_{0}\right)+2 p\left(z_{0}\right) z_{0} p^{\prime}\left(z_{0}\right)-2 p\left(z_{0}\right)-z_{0} p^{\prime}\left(z_{0}\right)}{2 p\left(z_{0}\right)+z_{0} p^{\prime}\left(z_{0}\right)}\right| \\
& =\left|\frac{2 M e^{i \theta}+2 M^{2} e^{2 i \theta}+m M e^{i \theta}+2 m M^{2} e^{2 i \theta}}{2+(2+m) M e^{i \theta}}\right| \\
& =\left|M e^{i \theta}\right| \cdot\left|1+\frac{m\left(1+M e^{i \theta}\right)}{2+(2+m) M e^{i \theta}}\right| \\
& =M\left|1+\frac{m\left(1+M e^{i \theta}\right)}{2+(2+m) M e^{i \theta}}\right| .
\end{aligned}
$$


In order to obtain the contradiction it suffices to show that the last expression is greater or equal to $M$ that is equivalent to the fact

$$
\left|1+\frac{m\left(1+M e^{i \theta}\right)}{2+(2+m) M e^{i \theta}}\right| \geq 1
$$

or

$$
\left|2+m+2(m+1) M e^{i \theta}\right|^{2} \geq\left|2+(2+m) M e^{i \theta}\right|^{2}
$$

The last inequality holds by virtue of the inequality

$M^{2}(3 m+4)+4(2+m) M \cos \theta+m+4 \geq M^{2}(3 m+4)-4(2+m) M+m+4 \geq 0$

or

$$
(3 m+4)(M-1)\left(M-\frac{4+m}{4+3 m}\right) \geq 0 .
$$

Since $M$ does not exceed $\frac{1}{3}$ the above expression is positive for every $m \geq 1$. This contradicts the hypothesis of the theorem, therefore $p \prec q$ and the proof of Theorem 2.5 is complete.

Let $p(z)=\frac{f(z)}{z}$. Then the previous theorem reduce to the following corollary:

Corollary 2.5 Let $f(z)=z+a_{2} z^{2}+\cdots$ be analytic in $\mathbb{D}$, and let $0<M<1$. Then

$$
\left|\frac{2 f^{\prime}(z)}{2+\frac{z f^{\prime}(z)}{f(z)}}-1\right|<M \Rightarrow\left|\frac{f(z)}{z}-1\right|<M
$$

For the case, when $p(z)=f^{\prime}(z)$, the Theorem 2.5 gives

Corollary 2.6 Let $f(z)=z+a_{2} z^{2}+\cdots$ be analytic in $\mathbb{D}$, and let $0<M<1$. Then

$$
\left|\frac{2\left(f^{\prime}(z)+z f^{\prime \prime}(z)\right)}{2+\frac{z f^{\prime \prime}(z)}{f^{\prime}(z)}}-1\right|<M \Rightarrow\left|f^{\prime}(z)-1\right|<M .
$$

Also, letting $p(z)=\frac{z f^{\prime}(z)}{f(z)}$ in Theorem 2.5, we conclude: 
Corollary 2.7 Let $f(z)=z+a_{2} z^{2}+\cdots$ be analytic in $\mathbb{D}$, and let $0<M<1$. Then

$$
\left|\frac{2 \frac{z f^{\prime}(z)}{f(z)}\left(2+\frac{z f^{\prime \prime}(z)}{f^{\prime}(z)}-\frac{z f^{\prime}(z)}{f(z)}\right)}{3+\frac{z f^{\prime \prime}(z)}{f^{\prime}(z)}-\frac{z f^{\prime}(z)}{f(z)}}-1\right|<M \Rightarrow\left|\frac{z f^{\prime}(z)}{f(z)}-1\right|<M .
$$

Theorem 2.6 Let $p(z)=1+a_{1} z+\cdots$ be analytic in $\mathbb{D}$ with $p(z) \not \equiv 1$ and let $\gamma \in(0,1]$. Then

$$
\left|\arg \frac{2 p(z)\left[p(z)+z p^{\prime}(z)\right]}{2 p(z)+z p^{\prime}(z)}\right|<\gamma \frac{\pi}{2} \Rightarrow|\arg p(z)|<\gamma \frac{\pi}{2} .
$$

Proof Let

$$
q(z)=\left(\frac{1+z}{1-z}\right)^{\gamma}=1+q_{1} z+\cdots,
$$

with $\Delta=q(\mathbb{D})=\left\{w:|\arg w|<\gamma \frac{\pi}{2}\right\}, q(0)=1, E(q)=\{1\}$, and $q \in Q$. Then, the condition (2.5) can be rewritten as

$$
\left|\arg \frac{2 p(z)\left[p(z)+z p^{\prime}(z)\right]}{2 p(z)+z p^{\prime}(z)}\right|<\gamma \frac{\pi}{2} \Rightarrow p(z) \prec q(z) .
$$

Suppose that $p(z) \nprec q(z)$.Then, from Lemma 1.1, there exist $z_{0} \in \mathbb{D}, \zeta_{0} \in \partial \mathbb{D} \backslash\{1\}$ and $m \geq 1$ such that $p\left(z_{0}\right)=q\left(\zeta_{0}\right)$ and $z_{0} p^{\prime}\left(z_{0}\right)=m \zeta_{0} q^{\prime}\left(\zeta_{0}\right)$. This implies that

$$
p\left(z_{0}\right)=q\left(\zeta_{0}\right):=(i x)^{\gamma}=x^{\gamma} e^{\gamma \frac{\pi}{2} i}
$$

where $x$ is a real number. Due to symmetry, it is sufficient to consider only the case where $x>0$. We have

$$
\zeta_{0}=q^{-1}\left(p\left(z_{0}\right)\right)=\frac{p\left(z_{0}\right)^{\frac{1}{\gamma}}-1}{p\left(z_{0}\right)^{\frac{1}{\gamma}}+1}
$$

and therefore we obtain

$$
z_{0} p^{\prime}\left(z_{0}\right)=m \zeta_{0} q^{\prime}\left(\zeta_{0}\right)=m \gamma x^{\gamma}\left(\frac{x^{2}+1}{2 x}\right) e^{\frac{\pi}{2}(\gamma+1) i}
$$


Taking into consideration (2.7) and (2.8), we have

$$
\begin{aligned}
\left|\arg \frac{2 p\left(z_{0}\right)\left[p\left(z_{0}\right)+z_{0} p^{\prime}\left(z_{0}\right)\right]}{2 p\left(z_{0}\right)+z_{0} p^{\prime}\left(z_{0}\right)}\right| & =\mid \arg \frac{2 p\left(z_{0}\right)\left[1+\frac{z_{0} p^{\prime}\left(z_{0}\right)}{p\left(z_{0}\right)}\right]}{2+\frac{z_{0} p^{\prime}\left(z_{0}\right)}{p\left(z_{0}\right)} \mid} \\
& =\left|\arg p\left(z_{0}\right)+\arg \frac{1+\frac{z_{0} p^{\prime}\left(z_{0}\right)}{p\left(z_{0}\right)}}{2+\frac{z_{0} p^{\prime}\left(z_{0}\right)}{p\left(z_{0}\right)}}\right| \\
& =\left|\gamma \frac{\pi}{2}+\arg \frac{1+u i}{2+u i}\right| \\
& =\left|\gamma \frac{\pi}{2}+\arctan \frac{u}{2+u^{2}}\right| \\
& \geq \gamma \frac{\pi}{2},
\end{aligned}
$$

where

$$
u=m \gamma\left(\frac{x^{2}+1}{2 x}\right)>0 .
$$

This contradicts the hypothesis of the theorem, therefore $p \prec q$ and the proof of Theorem 2.1 is complete.

Setting $p(z)=\frac{f(z)}{z}$ we obtain the following corollary:

Corollary 2.8 Let $f(z)=z+a_{2} z^{2}+\cdots$ be analytic in $\mathbb{D}$. Then

$$
\left|\arg \frac{2 f(z) f^{\prime}(z)}{f(z)+z f^{\prime}(z)}\right|<\gamma \frac{\pi}{2} \Rightarrow\left|\arg \frac{f(z)}{z}\right|<\gamma \frac{\pi}{2} .
$$

Theorem 2.7 Let $p(z)=1+a_{1} z+\cdots$ be analytic in $\mathbb{D}$ with $p(z) \not \equiv 1$ and let $\gamma \in(0,1]$. Then

$$
\left|\arg \frac{2\left[p(z)+\frac{z p^{\prime}(z)}{p(z)}\right]}{2+\frac{z p^{\prime}(z)}{p^{2}(z)}}\right|<\gamma \frac{\pi}{2} \Rightarrow|\arg p(z)|<\gamma \frac{\pi}{2}
$$

Proof We only need to show that $p \prec q$, where $q$ is given by (2.6). As in the proof of Theorem 2.6, there exist a point $z_{0} \in \mathbb{D}$ such that the equalities (2.7) and (2.8) holds. We have 


$$
\begin{aligned}
& \left|\arg \frac{2\left[p\left(z_{0}\right)+\frac{z_{0} p^{\prime}\left(z_{0}\right)}{p\left(z_{0}\right)}\right]}{2+\frac{z_{0} p^{\prime}\left(z_{0}\right)}{p^{2}\left(z_{0}\right)}}\right|=\left|\arg p\left(z_{0}\right)+\arg \frac{1+\frac{z_{0} p^{\prime}\left(z_{0}\right)}{p^{2}\left(z_{0}\right)}}{2+\frac{z_{0} p^{\prime}\left(z_{0}\right)}{p^{2}\left(z_{0}\right)}}\right| \\
& =\mid \arg (x i)^{\gamma}+\arg \left[\frac{2 x^{2 \gamma}+u^{2}+3 u x^{\gamma} \sin \gamma \frac{\pi}{2}}{4 x^{2 \gamma} \cos ^{2} \gamma \frac{\pi}{2}+\left(u+2 x^{\gamma} \sin \gamma \frac{\pi}{2}\right)}\right. \\
& \left.+i \frac{u x^{\gamma} \cos \gamma \frac{\pi}{2}}{4 x^{2 \gamma} \cos ^{2} \gamma \frac{\pi}{2}+\left(u+2 x^{\gamma} \sin \gamma \frac{\pi}{2}\right)}\right] \mid \text {, }
\end{aligned}
$$

where $u=m \gamma\left(\frac{x^{2}+1}{2 x}\right)>0$.

We notice that for $\gamma \in(0,1]$ the trigonometric functions sin and cos are located in the first quadrant of trigonometric circle and, therefore, have positive values. It follows that

$$
\left|\arg \frac{2\left[p\left(z_{0}\right)+\frac{z_{0} p^{\prime}\left(z_{0}\right)}{p\left(z_{0}\right)}\right]}{2+\frac{z_{0} p^{\prime}\left(z_{0}\right)}{p^{2}\left(z_{0}\right)}}\right|=\left|\gamma \frac{\pi}{2}+\arctan \frac{u \cos \gamma \frac{\pi}{2}}{2 x^{\gamma}+u^{2} x^{-\gamma}+3 u \sin \gamma \frac{\pi}{2}}\right| \geq \gamma \frac{\pi}{2},
$$

and this is contradiction with the hypothesis of the theorem. Thus, the proof is complete.

Setting $p(z)=\frac{z f^{\prime}(z)}{f(z)}$ we obtain

Corollary 2.9 Let $f(z)=z+a_{2} z^{2}+\cdots$ be analytic in $\mathbb{D}$. Then

$$
\left|\arg \frac{2 \frac{z f^{\prime}(z)}{f(z)}\left[1+\frac{z f^{\prime \prime}(z)}{f^{\prime}(z)}\right]}{1+\frac{z f^{\prime}(z)}{f(z)}+\frac{z f^{\prime \prime}(z)}{f^{\prime}(z)}}\right|<\gamma \frac{\pi}{2} \Rightarrow\left|\arg \frac{z f^{\prime}(z)}{f(z)}\right|<\gamma \frac{\pi}{2} .
$$

Acknowledgments This work was possible with the financial support of the Sectoral Operation Programme for Human Resources Development 2007-2013, co-financed by the European Social Fund, under the project number POSDRU/107/1.5/S/76841 with the title "Modern Doctoral Studies: Internationalization and Interdisciplinarity". This work was partially supported by the Centre for Innovation and Transfer of Natural Sciences and Engineering Knowledge, Faculty of Mathematics and Natural Sciences, University of Rzeszow.

\section{References}

1. Ali R.M., Nagpal S., Ravichandran, V.: Second-order differential subordination for analytic functions with fixed initial coefficient. Bull. Malays. Math. Sci. Soc. (2) 34(3), 611-629 (2011) 
2. Kanas S., Lecko A., Stankiewicz J.: Differential subordinations and geometric means. Compl. Var. 44. Theor. Appl. 28, 201-209 (1996)

3. Kanas S., Stankiewicz J.: Arithmetic means with reference to convexity conditions, Bull. Soc. Sci. Lett. Łódź 47, Ser. Rech. Deform. 24, 73-82 (1997)

4. Kanas, S., Lecko, A.: Univalence criteria connected with arithmetic and geometric means, II. Folia Sci. Univ. Tech. Resov. 20, 49-59 (1996)

5. Kanas S., Lecko A.: Univalence criteria connected with arithmetic and geometric means, II. In: Proceedings of the Second International Workshop of Transform Methods and Special Functions, Varna '96, pp. 201-209. Bulgarian Academy of Sciences, Sofia (1996)

6. Kim, Y.Ch., Lecko, A.: On differential subordinations related to convex functions. J. Math. Anal. Appl. 235, 130-141 (1999)

7. Lecko A., Lecko M.: Differential subordinations of arithmetic and geometric means of some functionals related to a sector. Int. J. Math. Math. Sci. 2011, Article ID 205845, 19 (2011)

8. Lewandowski, Z., Miller, S.S., Złotkiewicz, E.: Generating functions for some classes of univalent functions. Proc. Am. Math. Soc. 56, 111-117 (1976)

9. Liu J.-L.: Certain sufficient conditions for strongly starlike functions associated with an integral operator. Bull. Malays. Math. Sci. Soc. (2) 34(1), 21-30 (2011)

10. Miller, S.S., Mocanu, P.T.: Differential Subordinations: Theory and Applications. Dekker, New York (2000)

11. Mocanu, P.T.: Une propriété de convexité dans la théorie de représentation conforme. Stud. Univ. Babes-Bolyai 34(11), 127-134 (1969)

12. Mondal S.R., Swaminathan A.: Geometric properties of generalized Bessel functions. Bull. Malays. Math. Sci. Soc. (2) 35(1), 179-194 (2012)

13. Omar, R., Halim S.A.: Multivalent harmonic functions defined by Dziok-Srivastava operator. Bull. Malays. Math. Sci. Soc. (2) 35(3), 601-610 (2012)

14. Răducanu, D., Nechita, V.O.: On $\alpha$-convex analytic functions defined by generalized Ruscheweyh operator. Stud. Univ. Babeş-Bolyai 53, 109-118 (2008)

15. Ruscheweyh, S.: New criteria for univalent functions. Proc. Am. Math. Soc. 49, 109-115 (1975)

16. Ali, R.M., Ravichandran, V.: Classes of meromorphic $\alpha$ - convex functions. Taiwan. J. Math. 14, 1479$1490(2010)$

17. Supramaniam, S., Ali R.M., Lee S.K., Ravichandran, V.: Convolution and differential subordination for multivalent functions. Bull. Malays. Math. Sci. Soc. (2) 32(3), 351-360 (2009)

18. Sălăgean, G.: Subclasses of Univalent Functions, Lecture Notes in Math, vol. 1013. Springer, Berlin (1983) 